\title{
ORIGINAL ARTICLE \\ Preliminary study for the assessment of physical activity using a triaxial accelerometer with a gyro sensor on the upper limbs of subjects with paraplegia driving a wheelchair on a treadmill
}

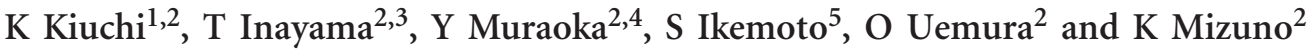

\begin{abstract}
Objective: This study aimed to examine whether, on the basis of the relationship between sensors attached on the upper limbs and energy expenditure (EE) at the time of wheelchair propulsion, there are differences in the measurement of $E E$ depending on the sensor attachment site and whether addition of the angular velocity information to the acceleration value is advantageous. We also aimed to clarify the variables used to estimate EE as well as the estimated error.

Setting: Laboratory of the National Hospital Organization Murayama Medical Center, Japan.

Methods: Six male subjects with spinal cord injuries participated in the study. Each wore sensors at the wrist and the middle upper arm on both sides while driving a wheelchair on a treadmill at three levels: very, very light; very light; and fairly light. Triaxial acceleration, triaxial angular velocity and EE were measured during driving. We analyzed the correlation between EE and acceleration, angular velocity and synthesized values of acceleration and angular velocity at each location using regression, multiple regression and Bland-Altman analyses.
\end{abstract}

Results: The determination coefficients between $\mathrm{EE}$ and the acceleration, angular velocity and synthesized values of acceleration and angular velocity varied from 0.68 to 0.87 at each location. The mean difference between the measured and estimated EE varied from 0.0028 (s.d., 0.0027 ) $\mathrm{kcal} \mathrm{min}^{-1} \mathrm{~kg}^{-1}$ on the right upper arm.

Conclusion: These findings suggest that combining the synthesized values of angular velocity and acceleration of the motion sensors on the upper limbs might reflect EE during a wheelchair driving activity on a treadmill.

Spinal Cord (2014) 52, 556-563; doi:10.1038/sc.2014.70; published online 13 May 2014

\section{INTRODUCTION}

Individuals with spinal cord injury (SCI) are prone to disuse syndrome due to their injury and limited movement. Compared with healthy people, people with SCI are at higher risk for lifestylerelated diseases and metabolic syndrome. ${ }^{1}$ Thus, to improve and maintain health, a plan for managing physical activity is necessary., ${ }^{2,3}$ A recent prospective cohort study showed that an increased physical activity level is associated with both improved physical fitness and a reduced risk of developing cardiovascular diseases. ${ }^{3}$ Devices are needed to assess physical activity and evaluate the efficacy of health-promotion interventions.

It is difficult to assess daily physical activity in community-dwelling people according to measurements of energy expenditure (EE) using criterion methods such as calorimetry or doubly labeled water techniques. Therefore, the development and validation of wearable monitors such as pedometers, load transducers/foot-contact monitors, accelerometers, heart rate monitors, combined accelerometer and heart rate monitors and multiple sensor systems that enable the evaluation of physical activity in healthy individuals are in progress. ${ }^{4}$
In Japan, Tanaka et al. ${ }^{5-7}$ investigated the relationships between each axes in a wearable lumbar triaxial accelerometer and EE in abled-bodied person, linear/non-linear relationship, adoption of a synthesized value and development of a gravity-removal classification algorithm; this has led to commercialization of the latter The validation of such diverse products contributes enormously to the research and development of practical activities for promoting the health of all people from children to the elderly. ${ }^{4,8}$

A series of reports based on studies of activity evaluation devices in wheelchair-using persons after SCI has been published in recent years. ${ }^{9-15}$ Coulter et al. ${ }^{9}$ and Sonenblum et al..$^{10,11}$ conducted several studies using multiaxial or triaxial accelerometers mounted on the wheels of wheelchairs. However, if the work involves mainly tasks using active muscles in the upper limbs, conducting an evaluation of physical activity using devices reflecting the movements of the upper limbs would be both legitimate and rational.

Among studies using activity monitors worn by participants on their wrists, Washburn and Copay ${ }^{16}$ examined the relationship between a uniaxial accelerometer count and $\mathrm{EE}$ during wheelchair

${ }^{1}$ Graduate School of Humanities and Sciences, Ochanomizu University, Bunkyo, Tokyo, Japan; ${ }^{2}$ Clinical Research Center, National Hospital Organization Murayama Medical Center, Musashimurayama, Tokyo, Japan; ${ }^{3}$ Department of Health Promotion Sciences, Graduate School of Human Health Sciences, Tokyo Metropolitan University, Hachioji, Tokyo, Japan; ${ }^{4}$ Faculty of Human Sciences, Waseda University, Tokorozawa, Saitama, Japan and ${ }^{5}$ Department of Human Nutrition, Seitoku University, Matsudo, Chiba, Japan Correspondence: Dr T Inayama, Department of Health Promotion Sciences, Graduate School of Human Health Sciences, Tokyo Metropolitan University, 1-1 Minami-Osawa, Hachioji-city, Tokyo 192-0397, Japan.

E-mail: tinayama@tmu.ac.jp

Received 31 January 2013; revised 17 March 2014; accepted 6 April 2014; published online 13 May 2014 
use. Warms et al. ${ }^{17,18}$ examined the relationship among a dual-axial accelerometer, actigraphy, the accelerometer's count values and each participant's self-reported activity levels. However, these reports were limited by the fact that they consisted of studies using acceleration values on a single axis ${ }^{16}$ and comparisons with self-reported activity levels. ${ }^{17}$ Tanhoffer et al. ${ }^{12}$ reported that the relationship between EE using the doubly labeled water technique and the predicted EE using multisensor armbands (SenseWear Armband, BodyMedia, Inc., Pittsburgh, USA) was $r^{2}=0.65(P<0.001)$ when the daily EE was evaluated but was $r^{2}=0.16(P=0.159)$ when the EE during activity was evaluated. In a study using a multisensor-based activity monitor (SensWear, BodyMedia, Inc., Pittsburgh, USA) attached to the upper arms of participants charged with resting, wheelchair propulsion, an arm-ergometer exercise and deskwork, Hiremath et al. ${ }^{13,14}$ reported the average EE estimation error based on the 'manufacturer's model' for estimating EE. Either way, for evaluating the physical activity of wheelchair users using activity monitors, more evidence will need to be gathered in future studies. However, the development of such devices has not progressed neither in Japan nor in other countries. In addition, because subjects with SCI have a low level of physical activity and $\mathrm{EE},{ }^{19-21}$ health promotion through the development and practical use of devices for assessing the physical activity of wheelchair users in their daily lives is required.

Figure 1 shows the directions of the three axes when the motion sensor used in this study was attached to the subjects' upper arms and the wrists. The dimension represented by the axis changes was dependent on the angle of the shoulder and elbow joints. In the movement of the shoulders, elbows and wrists, where the range of motion is wide, angular velocity and acceleration are involved. We hypothesize that a combination of the accelerometer or synthesized axes value as well as the angular velocity information may improve the estimation accuracy of these evaluation devices that reflect complex movements of the upper limbs.

This was a preliminary study that preceded the testing of the hypothesis mentioned above. A manual wheelchair driving load test using different intensities was performed for subjects with SCI by placing them on a treadmill. The purpose of this study was to examine, on the basis of the relationship between the EE and the acceleration and angular velocity values at the three axes attached to the upper limbs (left and right wrists, left and right upper arms) at the time of the wheelchair propulsion, whether there were differences

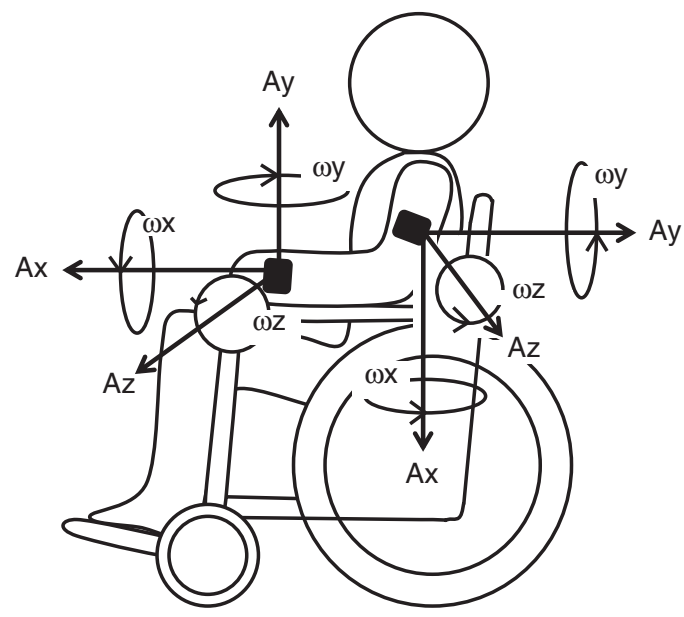

Figure 1 The triaxial accelerometer with a gyro sensor at the upper arms. A, Acceleration; $\omega$, angular velocity. depending on the sensor's attachment site and whether it is advantageous to add angular velocity information to the acceleration value. Using multiple regression analysis, we also aimed to clarify the variables used in the equation for estimating EE, the explanatory power and the size of the estimated error. Considering the merits and limitations of this study using loading with a single motion, future studies may use motion sensors for assessment of the physical activities of daily life.

\section{SUBJECTS AND METHODS}

\section{Subjects}

Six male subjects with SCI who routinely used a manual wheelchair participated in this study. The study was conducted according to the guidelines laid down in the Declaration of Helsinki, and all procedures involving human subjects were approved by the Ethics Committee of the National Hospital Organization Murayama Medical Center in Tokyo, Japan. The subjects had not participated in regular intensive physical exercise for the past year. The mean age (s.d.) was 34 years (s.d., 15), and the time since injury was 5.2 years (s.d., 6.7; range, 1-17.7 years). Injury levels were C6-C7, American Spinal Injury Association Impairment Scale grade (AIS) A in three subjects and T1-T9, AIS $\mathrm{A}$ in the others. All subjects were right handed. The study purpose and procedures were explained in detail to each subject before participation and all subjects provided informed consent. The descriptive characteristics of the study subjects are presented in Table 1 .

\section{Anthropometry}

Before the study, we measured each subject's height, body weight and left upper arm length. For measurement of height, the length from the parietal region to the heel was measured in the supine position. For measurement of body weight, the weight of the body and wheelchair was measured using a scale for wheelchairs, and the weights of the wheelchair and clothing were deducted from the subject's total weight. Body mass index was calculated as body weight $(\mathrm{kg})$ divided by the height squared $\left(\mathrm{m}^{2}\right)$. Upper arm length was measured from the acromion angle to the middle fingertip. We interviewed the subjects and recorded the time that had elapsed since the injury as well as the injury level.

\section{Experimental protocol}

Each subject drove a manual wheelchair on a treadmill. We used a bilaterally symmetrical and single-style motion consisting of propelling a wheelchair forward because if there were differences according to attachment site, it would be difficult to determine whether these differences were due to a characteristic property involving sensing of movements of the upper limbs, or whether they were due to motion patterns associated with asymmetric movements. The treadmill was designed for safe wheelchair driving, without derailing while being operated at a constant speed. The wheelchair was self-propelled and used in rehabilitation or daily activities.

Subjects completed the incremental load test at three levels. Because the participants had a wide age range (22-61 years) and the degree of physiological load is predicted to differ even at the same driving speed, the exercise intensity

Table 1 Descriptive characteristics of participants

\begin{tabular}{lrrr}
\hline & & \multicolumn{2}{c}{$\mathrm{n}=6$} \\
\cline { 3 - 4 } & & Mean & s.d. \\
\hline Age & Years & 34.3 & 15.0 \\
Number of years after injury & Years & 5.2 & 6.7 \\
Height & $\mathrm{cm}$ & 170.7 & 1.9 \\
Weight & $\mathrm{kg}$ & 62.7 & 11.9 \\
Body mass index & $\mathrm{kg} \mathrm{m}^{-2}$ & 21.5 & 3.8 \\
Upper limb length & $\mathrm{cm}^{-2}$ & 74.2 & 1.1 \\
\hline
\end{tabular}


was determined on the basis of the Rating of Perceived Exertion (RPE) scale. ${ }^{22}$ The participants maneuvered their wheelchairs continuously without pausing. In daily life, activities involving continuous maneuvering of a wheelchair are uncommon. In addition, none of the participants had the habit of practicing strenuous physical exercise. Therefore, the exercise intensity was not set to 'highly intense,' but rather to 'very, very light,' 9; 'very light,' 11; and 'fairly light,' 13. In a study that preceded the measurements, ${ }^{23}$ the subjects found it difficult to drive continuously for $9 \mathrm{~min}$; therefore, the duration of the wheelchair propelling exercise (driving time) was set to $2 \mathrm{~min}$ for each speed, corresponding to each intensity for a total of $6 \mathrm{~min}(2 \mathrm{~min} \times 3$ stages $)$.

On the day the measurements were obtained, the participants came to the measurement room $2 \mathrm{~h}$ after eating, as requested by us. Each participant put on a face mask, remained seated in a wheelchair and rested for $30 \mathrm{~min}$ before the EE measurement. Later, a driving test was performed on a treadmill designed for wheelchairs and set to driving speeds corresponding to RPE 9, RPE 11 and RPE 13 intensities. Thereafter, sensors were attached to the participants' left and right wrists as well as to their upper arms. After a 20-min pause, the participants wore face masks and maneuvered their wheelchairs at speeds that corresponded to each of the intensities. The gas analyzer that we used in this study displayed the gas volume, $\mathrm{RQ}, \mathrm{O}_{2}$ consumption, $\mathrm{CO}_{2}$ output and calculated $\mathrm{EE}$ value on a computer screen in real time every $15 \mathrm{~s}$. Visual confirmation was performed on the screen to check if the EE consumption had increased and reached a steady state; in each of the 2 min driving durations at each stage, the EE during the latter half $(1 \mathrm{~min})$ as well as the values of acceleration and angular velocity were used in the analysis. The subjects were permitted to consume only drinking water during the experiment.

\section{EE measurements}

Although the subjects wore a Rudolf face mask covering their mouth and nose, the expired gas was sampled by the gas analyzer (AR-1 Type-4; Arco System, Chiba, Japan) with a galvanic $\mathrm{O}_{2}$ sensor and an infrared $\mathrm{CO}_{2}$ sensor. Concentrations and volumes of expired $\mathrm{O}_{2}$ and $\mathrm{CO}_{2}$ were measured. For each measurement, the gas analyzer was initially calibrated using a certified gas mixture and atmospheric air. The gas analyzer (AR-1 type-4) has been proven both reliable and valid in the general population. ${ }^{24}$ The values of $\mathrm{O}_{2}$ consumption and $\mathrm{CO}_{2}$ production were expressed under standard temperature, pressure and dry air conditions. EE was estimated from $\mathrm{O}_{2}$ consumption and $\mathrm{CO}_{2}$ production using Weir's equation. ${ }^{25}$ Metabolic equivalent (MET) values as a reference were calculated as follows: EE during the driving divided by the measured $\mathrm{EE}$ at rest for $10 \mathrm{~min}$.

\section{Measurement of acceleration and angular velocity}

We measured acceleration and angular velocity using a motion sensor including a triaxial accelerometer with a gyro sensor (MicroStone Corporation, Nagano, Japan). The sensor was built into a plastic case without a liquid crystal display and was designed to be worn on the arm (size, $45 \times 45 \times 23 \mathrm{~mm}$; weight, approximately $55 \mathrm{~g}$ ). Anteroposterior ( $x$-axis), vertical ( $y$-axis) and mediolateral ( $z$-axis) acceleration and gyro measurements were obtained at a rate of $200 \mathrm{~Hz}$. The sensor was attached at two locations on each side: (1) the dorsal side of the distal end of the forearm and (2) the midpoint between the acromion angle and the olecranon (hereinafter referred to as left wrist, right wrist, left upper arm and right upper arm). Each axis on the wrist and upper arm is shown in Figure 1. The acceleration data were uploaded to a personal computer.

The signals obtained from the sensor were processed as follows. Each of the three signals from the triaxial accelerometer was passed through a high-pass filter with a cutoff frequency of $0.5 \mathrm{~Hz}$ to remove the gravitational acceleration component and sensor drift from the signal and through a low-pass filter with a cutoff frequency of $5 \mathrm{~Hz}$ to remove the acceleration of the hand colliding with the handrim during wheelchair operation. Each of the three signals from the gyro sensor was not passed through a filter. In our earlier preliminary study on wheelchair driving by able-bodied people, we reported that use of the filter was required to remove the effect of gravitational acceleration and the impact during wheelchair handling for the acceleration data, not the angular velocity data. ${ }^{26}$ The filtered acceleration and raw angular velocity data were calculated as the average of the absolute values in each direction.

\section{Statistical analyses}

EE and MET values are presented as means and s.d.. Differences between EE at different exercise intensity levels were assessed by one-way repeated-measures analysis of variance followed by Bonferroni's post hoc test. Relationships between measured $\mathrm{EE}$ and each motion sensor value were evaluated with coefficient of determination $\left(r^{2}\right)$ using regression analysis. Multiple regression analysis was performed to develop equations predicting EE. The independent variables were as follows: model 1: $\mathrm{A}(x), \mathrm{A}(y), \mathrm{A}(z), \mathrm{A}(x y), \mathrm{A}(x z), \mathrm{A}(y z)$ and $\mathrm{A}(x y z)$; model 2: $\omega(x), \omega(y), \omega(z), \omega(x y), \omega(x z), \omega(y z)$ and $\omega(x y z)$; model 3: acceleration, angular velocity and synthesized values for both and age, height, weight, upper arm length, injury level and time since the injury were used as independent variables in the stepwise method. The relationships between measured and estimated EE values from model three were examined using Bland-Altman analysis. Statistical analyses were carried out using IBM SPSS Statistics v. 21 (IBM Japan Inc., Tokyo, Japan). The level of statistical significance was set at $5 \%$ for the two-tailed tests.

\section{RESULTS}

\section{Wheelchair exercise}

The treadmill speed during the wheelchair exercise was $2.5-3.0 \mathrm{kmh}^{-1}$ at RPE 9, $3.5-4.0 \mathrm{kmh}^{-1}$ at RPE 11 and $4.5-5.0 \mathrm{~km} \mathrm{~h}^{-1}$ at RPE 13. The EE and MET values for each level are shown in Table 2 . The EE was increased in an intensity-dependent

Table 2 Energy expenditure and MET for each driving on a treadmill

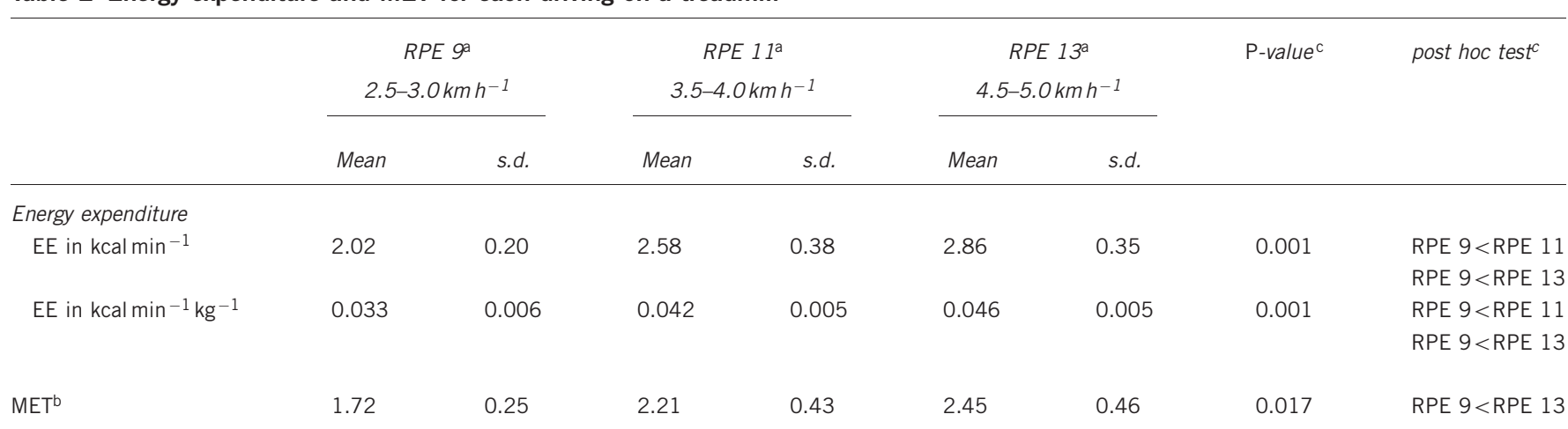

Abbreviations: $\mathrm{EE}$, energy expenditure; MET, metabolic equivalents; RPE, rating of perceived exertion.

a Subjects carried out the incremental load test at three levels on the RPE scale: RPE 9, very, very light; RPE 11, very light; RPE 13, fairly light.

bMET were calculated as EE for each driving divided by EE for resting in the sitting position. EE in the sitting position: $1717 \pm 265 \mathrm{kcal}$ per day.

'Differences between $\mathrm{EE}$ in different levels of exercise intensity were assessed by one-way repeated-measures analysis of variance followed by Bonferroni's post hoc test. 
manner (RPE 9 vs RPE $11, P<0.05$; RPE 9 vs RPE 13, $P<0.01$ ), although there was no significant difference between RPE 11 and RPE 13. Mean MET was 1.72 at RPE 9, 2.21 at RPE 11 and 2.45 at RPE 13.

\section{Acceleration and angular velocity during wheelchair exercise}

Mean acceleration, angular velocity and synthesized values of both for each wheelchair driving level as well as their relationships with measured EE are shown in Table 3. The coefficient between EE and each acceleration was distributed over a wide range by sensor location $\left(r^{2}=0.16-0.82\right)$, EE and each angular velocity $\left(r^{2}=0.05-0.64\right)$. The synthesized value for three-axis acceleration, $\mathrm{A}(x y z)$, showed $r^{2}=0.55$ $(P<0.001)$ or more on every sensor location. In this study, analyses were performed using linear, logarithmic and quadratic regressions. The results showed that the determination coefficient per measurement site was comparable for all of the regression methods (Table 3).

\section{Estimated EE during wheelchair exercise}

For the motion sensors used in this study, the values found at the single $x, y$ and $z$ axes as well as the resultant of the values thereof consisted of 7 values for the acceleration and angular velocity. The degree of influence of these independent variables was analyzed at the time of use of $\mathrm{EE}$ as a dependent variable, and multiple regression analysis was performed to examine the prediction equation (Table 4). The determination coefficient with EE varied from 0.64 to 0.82 (standard error of the estimation (SEE), 0.003 to 0.005 ) in model 1 using the acceleration data, $0.50-0.83$ (SEE, 0.003 to 0.005 ) in model 2 using the angular velocity and 0.68 to 0.87 (SEE, 0.003 to 0.004 ) in model 3 using a combination of acceleration and angular velocity. Figure 2 further illustrates the relationship between the measured EE and the estimated $\mathrm{EE}$ on the multiple regression equations (model 3) using Bland-Altman analysis. The mean difference between the estimated and measured EE was -0.0026 (s.d., 0.0029) $\mathrm{kcal} \mathrm{min}^{-1}$ $\mathrm{kg}^{-1}$ on the left wrist, -0.0036 (s.d., 0.0042 ) $\mathrm{kcal} \mathrm{min}^{-1} \mathrm{~kg}^{-1}$ on the right wrist, 0.0011 (s.d., 0.0038 ) $\mathrm{kcal} \mathrm{min}^{-1} \mathrm{~kg}^{-1}$ on the left upper arm and 0.0028 (s.d., 0.0027 ) $\mathrm{kcal} \mathrm{min}^{-1} \mathrm{~kg}^{-1}$ on the right upper arm.

\section{DISCUSSION}

This study's findings showed that the combination of synthesized values of acceleration and angular velocity of the motion sensors attached to the upper limbs might reflect the EE during a wheelchair driving activity on a treadmill. The findings of multiple regression analyses imply that combined data pertaining to acceleration and angular velocity might be meaningful for the evaluation of upper limb movements. However, in symmetrical single-type movements consisting of driving straight and continuously on a wheelchair in a small sample size study, it was difficult to determine the optimal attachment sites. A more in-depth study of the differences associated with the attachment sites is required when the upper limbs are used as sensing sites.

At the time of the loading of treadmill driving using a wheelchair, the estimated MET was 1.7-2.5. The intensity corresponds to that of a healthy subject in the sitting position performing a muscular activity (MET, 1.8-2.5), stretching (MET, 2.3), yoga (MET, 2.5) or walking slowly at $3.2 \mathrm{~km} \mathrm{~h}^{-1}$ (MET, 2.8). ${ }^{27}$ Loading tests were performed using activity intensities estimated to be RPE 9, RPE 11 and RPE 13; however, based on the estimated MET, the load range was categorized as being of mild intensity.

Among the values of the coefficient of determination using linear, logarithmic and quadratic regression analyses at the attachment sites, the variables in the same axis did not necessarily show the same explanatory power in the left and right wrists or both upper arms (Table 3). The differences in the dominant hand and in each individual's method of maneuvering the wheelchair may be reflected in the data obtained from motion sensors attached to the upper limbs. Washburn and Copay ${ }^{16}$ reported that significant associations between a uniaxial accelerometer count and EE were $r^{2}=0.27$ for the right wrist and $r^{2}=0.44$ for the left wrist during wheelchair pushing at three different speeds. It is necessary to determine appropriate attachment sites for protocols using diversified exercise types. Reports have also shown that the correlation between $\mathrm{EE}$ and the motion sensor data is not necessarily linear, even during low-intensity activities. ${ }^{4,5}$ The loading used in the low-intensity exercise performed in this study suggested that there were no definite differences between linear and non-linear variations; therefore, studies using an additional high-intensity exercise load are needed in the future.

In multiple regression analyses using both acceleration and angular velocity data (model 3) as independent variables (Table 4), the determination coefficients varied from 0.68 to 0.87 , and the SEE range was narrow $(0.003-0.004)$. The Bland-Altman plots in Figure 2 showing the correlation between the estimated and measured EE values suggest that although overestimation is likely to occur, the estimation error is small. Several studies ${ }^{5-7}$ on healthy subjects with triaxial accelerometers attached to the waist support the usefulness of combined values. As shown in Figure 1, in motions involving complex movements of the shoulder, elbow and wrist joints, where the direction of the movement acceleration is not constant when viewed from the sensor's axis, use of synthesized data as variables is predicted to be more rational. In addition, Hiremath et al. ${ }^{14}$ reported that the average EE estimation error using general and activityspecific EE prediction models was $2.30 \%$ and $4.85 \%$, respectively; these models were based on SenseWear for four activities, namely, resting, wheelchair propulsion, arm-ergometer exercise and deskwork. SenseWear consisted of a two-axis accelerometer, a galvanic skin response sensor, a skin temperature sensor and a near-body temperature sensor. In this small sample size study, the results merely suggest that when an upper limb with a diverse range of movements is used as a sensing site, combining the synthesized acceleration and angular velocity values might be potentially useful for estimating the activity amount. Studies using protocols with a diversified motion style are required to verify this hypothesis.

In addition, the issue that needs to be considered regarding the variables in the multiple regression equation (Table 4 ) is the fact that at all sensor attachment sites, the elements of the $z$-axis, that is, $\mathrm{A}(z)$, $\mathrm{A}(x z)$ and $\mathrm{A}(x y z)$, were extracted from the acceleration data. In a single-style motion consisting of driving a wheelchair continuously in a straight and forward direction, the acceleration toward the lateral side shown in Figure 1 may reflect the properties of the upper limb.

This study has several limitations. The most important issues were that the number of participants was small and that the measurements were not obtained repeatedly. This preliminary study showed that repeated measurements using a greater sample size will be needed to verify experimental validity and reliability. The protocol used in this study, that is, the 6-min treadmill driving involving three levels of mild-intensity activity, also had its limitations with regard to activity type, duration and intensity. Driving a wheelchair continuously at a constant speed is not likely to happen frequently in daily life. As doing so, results in the occurrence of muscle fatigue associated with consecutive muscle contractions in the upper limbs, the protocol was set in a way that the driving time duration at each stage was as short as $2 \mathrm{~min}$. In addition, the participants in this study had no habit 


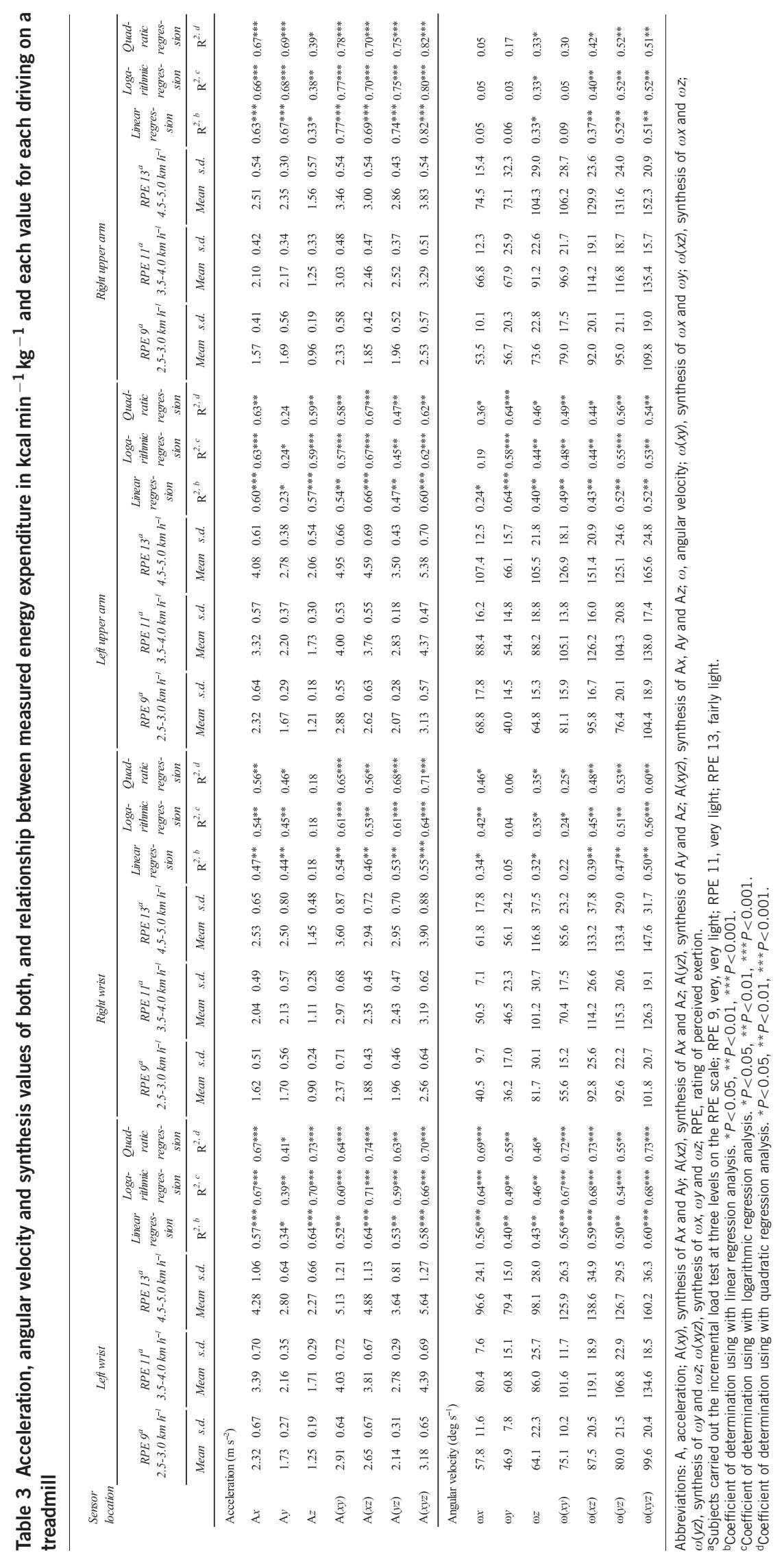


Table 4 Multiple regression analysis model for estimation of energy expenditure in $\mathrm{kcal} \mathrm{min}^{-1} \mathrm{~kg}^{-1}$

\begin{tabular}{|c|c|c|c|c|c|c|c|c|c|c|}
\hline \multirow[b]{2}{*}{ Sensor Location } & \multirow[b]{2}{*}{ Equation $^{\mathrm{a}}$} & \multirow[b]{2}{*}{$\mathrm{R}^{2}$} & \multirow[b]{2}{*}{ P-value } & \multirow[b]{2}{*}{ SEE } & \multicolumn{6}{|c|}{ Variables in the equation } \\
\hline & & & & & Variables & $\begin{array}{l}\text { Partial } \\
\text { regression } \\
\text { coefficient }\end{array}$ & s.e. & $\begin{array}{c}\text { Standardized } \\
\text { partial } \\
\text { regression } \\
\text { coefficient }\end{array}$ & $t$ & P-value \\
\hline \multicolumn{11}{|l|}{ Model $1^{\text {a }}$} \\
\hline \multirow[t]{2}{*}{ Right wrist } & $\begin{array}{l}\mathrm{EE}=0.2139+0.0072 \times \mathrm{A}(x y z)- \\
0.0027 \times \text { upper limb length }\end{array}$ & 0.68 & $<0.001$ & 0.004 & $A(x y z)$ & 0.007 & 0.001 & 0.855 & 5.61 & $<0.001$ \\
\hline & & & & & $\begin{array}{l}\text { upper } \\
\text { limb } \\
\text { length }\end{array}$ & -0.003 & 0.001 & -0.377 & -2.47 & 0.026 \\
\hline Left upper arm & $\mathrm{EE}=0.0185+0.00594 \times \mathrm{A}(x z)$ & 0.66 & $<0.001$ & 0.004 & $A(x z)$ & 0.006 & 0.001 & 0.813 & 5.58 & $<0.001$ \\
\hline Right wrist & $\mathrm{EE}=0.0182+0.0002 \times \omega(x y z)$ & 0.50 & 0.001 & 0.005 & $\omega(x y z)$ & 0.000 & 0.000 & 0.709 & 4.02 & 0.001 \\
\hline Left upper arm & $\mathrm{EE}=0.0224+0.0003 \times \omega(y)$ & 0.64 & $<0.001$ & 0.005 & $\omega(y)$ & 0.000 & 0.000 & 0.799 & 5.32 & $<0.001$ \\
\hline \multirow[t]{3}{*}{ Right upper arm } & $\begin{array}{l}\mathrm{EE}=-0.3084+0.0004 \times \omega(y z)+ \\
0.0029 \times \text { height }-0.0026 \times \\
\text { upper limb length }\end{array}$ & 0.83 & $<0.001$ & 0.003 & $\omega(y z)$ & 0.000 & 0.000 & 1.200 & 8.28 & $<0.001$ \\
\hline & & & & & height & 0.003 & 0.001 & 0.685 & 4.75 & $<0.001$ \\
\hline & & & & & $\begin{array}{l}\text { upper } \\
\text { limb } \\
\text { length }\end{array}$ & -0.003 & 0.001 & -0.363 & -3.20 & 0.006 \\
\hline \multicolumn{11}{|l|}{ Model $3^{c}$} \\
\hline Left wrist & $\begin{array}{l}\mathrm{EE}=0.0200+0.0110 \times \mathrm{A}(\mathrm{z})+ \\
0.0002 \times \omega(y z)-0.0101 \times \mathrm{A}(y)\end{array}$ & 0.86 & $<0.001$ & 0.003 & $A(z)$ & 0.011 & 0.002 & 0.865 & 5.86 & $<0.001$ \\
\hline \multirow[t]{2}{*}{ Left upper arm } & $\begin{array}{l}\mathrm{EE}=0.0175+0.0036 \times \mathrm{A}(x z)+ \\
0.0002 \times \omega(y)\end{array}$ & 0.75 & $<0.001$ & 0.004 & $A(x z)$ & 0.004 & 0.001 & 0.489 & 2.51 & 0.024 \\
\hline & & & & & $\omega(y)$ & 0.000 & 0.000 & 0.436 & 2.24 & 0.041 \\
\hline \multirow[t]{2}{*}{ Right upper arm } & $\begin{array}{l}\mathrm{EE}=0.0064+0.0079 \times \mathrm{A}(x y z)+ \\
0.0001 \times \omega(x z)\end{array}$ & 0.87 & $<0.001$ & 0.003 & $A(x y z)$ & 0.008 & 0.001 & 0.792 & 7.68 & $<0.001$ \\
\hline & & & & & $\omega(x z)$ & 0.000 & 0.000 & 0.257 & 2.49 & 0.025 \\
\hline
\end{tabular}

Abbreviations: $A$; acceleration; $A(x z)$, synthesis of $A x$ and $A z ; A(x y z)$, synthesis of $A x, A y$ and $A z ; E E$, energy expenditure; SEE, standard error of the estimation $\omega$, angular velocity; $\omega(x z)$, synthesis of $\omega x$ and $\omega z ; \omega(y z)$, synthesis of $\omega y$ and $\omega z ; \omega(x y z)$, synthesis of $\omega X, \omega y$ and $\omega z$.

aDependent variable: EE (kcal kg $\left.{ }^{-1} \mathrm{~min}^{-1}\right)$; independent variables: acceleration $\mathrm{A}\left(\mathrm{ms}^{-2}\right)$, synthesis values, age (years), height (cm), weight $(\mathrm{kg})$, upper limb length (cm), level of injury, time since injury (years).

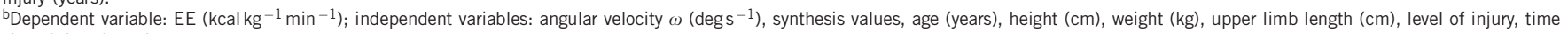
since injury (years).

cDependent variable: $\mathrm{EE}\left(\mathrm{kcal} \mathrm{kg}^{-1} \mathrm{~min}^{-1}\right)$; independent variables: acceleration $\mathrm{A}\left(\mathrm{ms}^{-2}\right.$ ), angular velocity $\omega$ (deg s${ }^{-1}$ ), synthesis values, age (years), height (cm), weight (kg), upper limb length $(\mathrm{cm})$, level of injury, time since injury (years).

of practicing intense physical exercise; therefore, no high-intensity activity was used in the loading experiments. High-intensity activities similar to those performed during physical exercise may need to be examined in the future. In addition, because the participants ran at a fixed location on a treadmill designed for wheelchairs, accelerations associated with normal movements did not occur.

For several activities involving a diverse range of movements similar to those actually found in daily life, various protocols are required for studying the optimal sites for the attachment of motion sensors, and the advantage of adopting synthesized values of acceleration/angular velocity and combining the angular velocity with acceleration. In addition, the acquisition of a sample size that enables the verification of differences due to injury levels remains a challenge. Wheelchair maneuvering differed according to factors such as injury site, upper limb muscle mass, the presence of paraplegia or quadriplegia and complete or incomplete injury, wheelchair shape and 
a
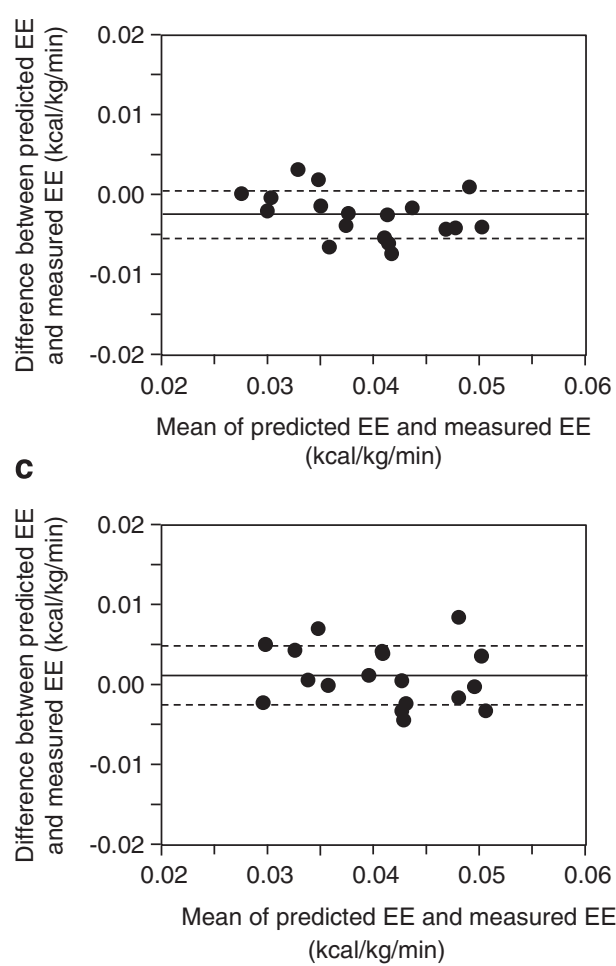

b
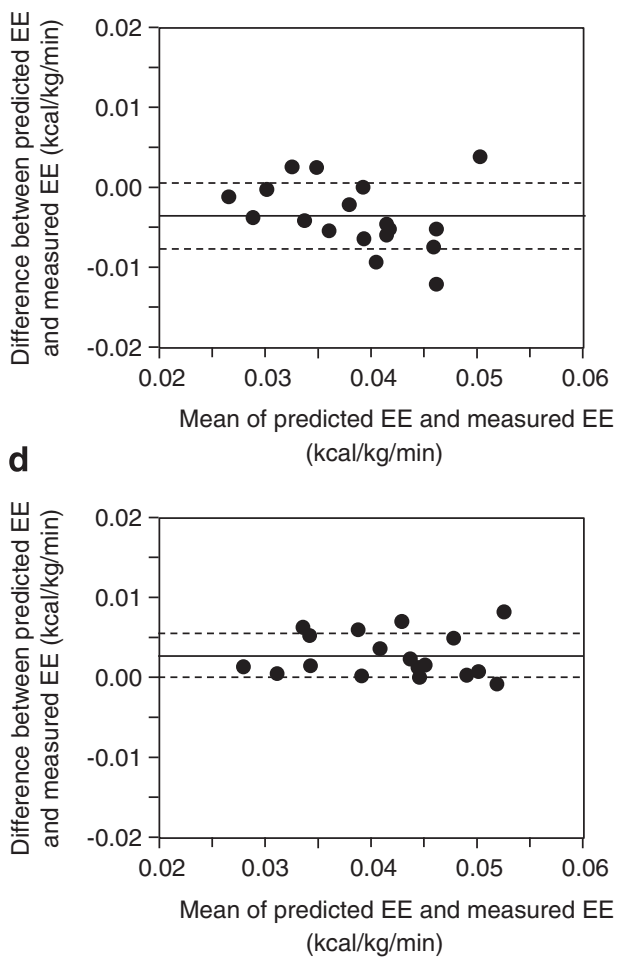

Figure 2 Bland-Altman analysis. The differences between predicted energy expenditure (EE) and measured EE were plotted against the mean of predicted $\mathrm{EE}$ and measured EE for driving a wheelchair. a, left wrist; b, right wrist; c: left upper arm; d, right upper arm. -, mean; ---, s.d.

floor surface. ${ }^{28,29}$ Hiremath et al. previously stated that for estimating $\mathrm{EE}$, subject body measurement parameters were also needed in addition to movement parameters. ${ }^{13,14}$ There is a need to integrate research, ${ }^{8}$ leading to a final evaluation of physical activity in daily life through verification of the validity and reliability of the activity estimation using a triaxial accelerometer with a gyro sensor. Among industrialized nations and regions, Japan has a low frequency of obesity, and its citizens are at low risk for developing heart disease. Because of the development of devices for evaluating physical activity, the evaluation of physical activity in subjects with SCI and wheelchair users may result in both a decreased risk of lifestyle-related diseases ${ }^{1}$ and additional findings pertaining to the correlation between physical activity and quality of life $\mathrm{e}^{30}$ in various regions with different backgrounds in terms of disease risk.

\section{CONCLUSION}

This study suggested that the combination of synthesized values of angular velocity and acceleration of the motion sensors attached to the upper limbs might reflect the EE during a wheelchair driving activity on a treadmill.

\section{DATA ARCHIVING}

There were no data to deposit.

\section{CONFLICT OF INTEREST}

The authors declare no conflict of interest.

\section{ACKNOWLEDGEMENTS}

We thank all the individuals who volunteered for this study.
1 Garshick E, Kelley A, Cohen SA, Garrison A, Tun CG, Gagnon D et al. A prospective assessment of mortality in chronic spinal cord injury. Spinal Cord 2005; 43: 408-416.

2 Ginis KA, Hicks AL, Latimer AE, Warburton DE, Bourne C, Ditor DS et al. The development of evidence-informed physical activity guidelines for adults with spinal cord injury. Spinal Cord 2011; 49: 1088-1096.

3 Nooijen CF, de Groot S, Postma K, Bergen MP, Stam HJ, Bussmann JB et al. A more active lifestyle in persons with a recent spinal cord injury benefits physical fitness and health. Spinal Cord 2012; 50: 320-323.

4 Butte NF, Ekelund U, Westerterp KR. Assessing physical activity using wearable monitors: measures of physical activity. Med Sci Sports Exerc 2012; 44: S5-S12.

5 Tanaka C, Tanaka S, Kawahara J, Midorikawa T. Triaxial accelerometry for assessment of physical activity in young children. Obesity (Silver Spring) 2007; 15: 1233-1241.

6 Midorikawa T, Tanaka S, Kaneko K, Koizumi K, Ishikawa-Takata K, Futami J et al. Evaluation of low-intensity physical activity by triaxial accelerometry. Obesity (Silver Spring) 2007; 15: 3031-3038.

7 Ohkawara K, Oshima Y, Hikihara Y, Ishikawa-Takata K, Tabata I, Tanaka S. Real-time estimation of daily physical activity intensity by a triaxial accelerometer and a gravityremoval classification algorithm. Br J Nutr 2011; 105: 1681-1691.

8 Intille SS, Lester J, Sallis JF, Duncan G. New horizons in sensor development. Med Sci Sports Exerc 2012; 44: S24-S31.

9 Coulter EH, Dall PM, Rochester L, Hasler JP, Granat MH. Development and validation of a physical activity monitor for use on a wheelchair. Spinal Cord 2011; 49: 445-450.

10 Sonenblum SE, Sprigle S, Harris FH, Maurer CL. Characterization of power wheelchair use in the home and community. Arch Phys Med Rehabil 2008; 89: 486-491.

11 Sonenblum SE, Sprigle S, Caspall J, Lopez R. Validation of an accelerometer-based method to measure the use of manual wheelchairs. Med Eng Phys 2012; 34: 781-786.

12 Tanhoffer RA, Tanhoffer AI, Raymond J, Hills AP, Davis GM. Comparison of methods to assess energy expenditure and physical activity in people with spinal cord injury. J Spinal Cord Med 2012; 35: 35-45.

13 Hiremath SV, Ding D. Evaluation of activity monitors in manual wheelchair users with paraplegia. J Spinal Cord Med 2011; 34: 110-117.

14 Hiremath SV, Ding D, Farringdon J, Cooper RA. Predicting energy expenditure of manual wheelchair users with spinal cord injury using a multisensory-based activity monitor. Arch Phys Med Rehabil 2012; 93: 1937-1943.

15 Harris F, Sprigle S, Sonenblum SE, Maurer CL. The participation and activity measurement system: an example application among people who use wheeled mobility devices. Disabil Rehabil Assist Technol 2010; 5: 48-57.

16 Washburn RA, Copay AG. Assessing physical activity during wheelchair pushing: validity of a portable accelerometer. APAQ 1999; 16: 290-299. 
17 Warms CA, Belza BL. Actigraphy as a measure of physical activity for wheelchair users with spinal cord injury. Nurs Res 2004; 53: 136-143.

18 Warms CA, Whitney JD, Belza B. Measurement and description of physical activity in adult manual wheelchair users. Disabil Health J 2008; 1: 236-244.

19 Monroe MB, Tataranni PA, Pratley R, Manore MM, Skinner JS, Ravussin E. Lower daily energy expenditure as measured by a respiratory chamber in subjects with spinal cord injury compared with control subjects. Am J Clin Nutr 1998; 68 1223-1227.

20 Buchholz AC, McGillivray CF, Pencharz PB. Physical activity levels are low in free-living adults with chronic paraplegia. Obes Res 2003; 11: 563-570.

21 Buchholz AC, Pencharz PB. Energy expenditure in chronic spinal cord injury. Curr Opin Clin Nutr Metab Care 2004; 7: 635-639.

22 Borg GA. Psychophysical bases of perceived exertion. Med Sci Sports Exerc 1982; 14 377-381.

23 Kiuchi K, Inayama T, Oda H, Tsunoda N, Oka J, Higuchi Y et al. Preliminary study for evaluation of energy expenditure on wheelchair movement by a wristwatch type triaxial accelerometer with triaxial gyrometer in able-bodied people. Jap J Clin Sports Med 2011; 19: 265-271. (in Japanese).
24 Tanaka S, Ohkawara K, Takata K, Morita A, Watanabe S. Accuracy of predictive equations for basal metabolic rate and contribution of abdominal fat distribution to basal metabolic rate in obese Japanese people. Anti-Aging Med 2008; 5: 17-21.

25 Weir JB. New methods for calculating metabolic rate with special reference to protein metabolism. J Physiol 1949; 109: 1-9.

26 Kiuchi K, Muraoka Y, Ikemoto S, Uemura O, Mizuno K, Wakatani Y et al. Evaluation of the amount of physical activities of a wheelchair driver driving on a treadmill by using a three dimensions accelerometer with a gyro sensor located on the upper extremities. Jap J Clin Sports Med 2012; 20: 578-585. (in Japanese).

27 Ainsworth BE, Haskell WL, Herrmann SD, Meckes N, Bassett DR Jr, Tudor-Locke C et al. 2011 Compendium of Physical Activities: a second update of codes and MET values. Med Sci Sports Exerc 2011; 43: 1575-1581.

28 Beekman CE, Miller-Porter L, Schoneberger M. Energy cost of propulsion in standard and ultralight wheelchairs in people with spinal cord injuries. Phys Ther 1999; 79: 146-158.

29 Sanderson DJ, Sommer HJ 3rd. Kinematic features of wheelchair propulsion. J Biomech 1985; 18: 423-429.

30 Martin Ginis KA, Jetha A, Mack DE, Hetz S. Physical activity and subjective well-being among people with spinal cord injury: a meta-analysis. Spinal Cord 2010; 48: 65-72. 